\title{
Enhancing and degrading visual stimuli
}

\author{
JOHN O. BROOKS III \\ Rice University, Houston, Texas
}

\begin{abstract}
Several assembly language routines are described for use in perceptual identification experiments on the Apple Macintosh computer with MS-BASIC. These routines are in a machine language library that may be accessed from BASIC. No knowledge of assembly language is required to use the statements. In one method of perceptual identification, stimulus clarification, a mask is gradually removed to reveal the stimulus behind it. The other method, stimulus materialization, displays the stimulus on the screen in a piecemeal fashion. Data are reported from a demonstration experiment in which prior exposure to words enabled subjects to identify those words more easily under impoverished conditions.
\end{abstract}

Measurements of perceptual identification have been used frequently to demonstrate the facilitation in performance resulting from previous experiences commonly referred to as priming effects. In the perceptual identification task, subjects are asked to identify a stimulus under visually degraded conditions. The general finding is that subjects are able to identify previously presented stimuli more rapidly than stimuli they have not seen before. This finding has been applied across a wide range of areas and in numerous situations. For example, Gollin (1960) used degraded images of line drawings to examine learning in children. Cermak, Talbot, Chandler, and Wolbarst (1985) applied the technique to the study of perceptual priming in amnesic patients. Johnston, Dark, and Jacoby (1985) employed a perceptual identification task with college students to assess priming for words and pseudowords.

This paper describes several assembly language routines that can be used to conduct experiments involving perceptual identification on the Macintosh using Microsoft BASIC (MS-BASIC, version 2.0 or later). The routines create masks and present degraded images of the stimulus on the screen. Although the routines are presented in the context of their use pertaining to perceptual identification, they may be applied to many other situations.

The routines allow the implementation of two variations of the perceptual identification task. In the first method, stimulus clarification, a random-dot noise pattern, or mask, is superimposed over the stimulus. The resulting degraded image is then clarified gradually by removing a specified portion of the mask at a rate that is either preset or paced by the subject. Clarification may end at a predetermined point or when the subject makes a response. Figure 1 illustrates three possible steps in the clarification of the word Ebbinghaus. The second method

This article is based on work supported by a National Science Foundation Graduate Fellowship. Requests for reprints may be addressed to the author at the Department of Psychology, Rice University, Houston, TX 77251 .

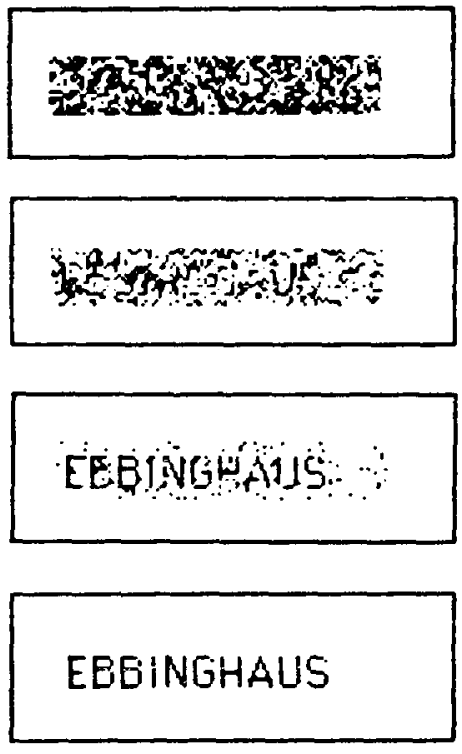

Figure 1. Three possible steps in the clarification of the word $E b$ binghaus.

of perceptual identification is stimulus materialization in which the stimulus gradually appears, pixel by pixel, on a blank screen. This procedure creates the illusion that the stimulus is materializing on the computer screen, as illustrated in Figure 2. As with stimulus clarification, stimulus materialization continues until a preset limit is reached or the subject makes a response.

Vokey, Baker, Hayman, and Jacoby (1986) provided several assembly language programs for the Apple II that can be used to study perceptual identification. The Macintosh, with its sophisticated microprocessor and highquality graphics, is also well suited for such research. Indeed, the standard formats for graphics on the Macintosh allow BASIC and other programming languages to access stimuli that have been created by other applications.

As implemented on the Macintosh, MS-BASIC gives programmers the ability to extend the BASIC language through the inclusion of their own assembly language routines. The routines are placed in what is known as a library 


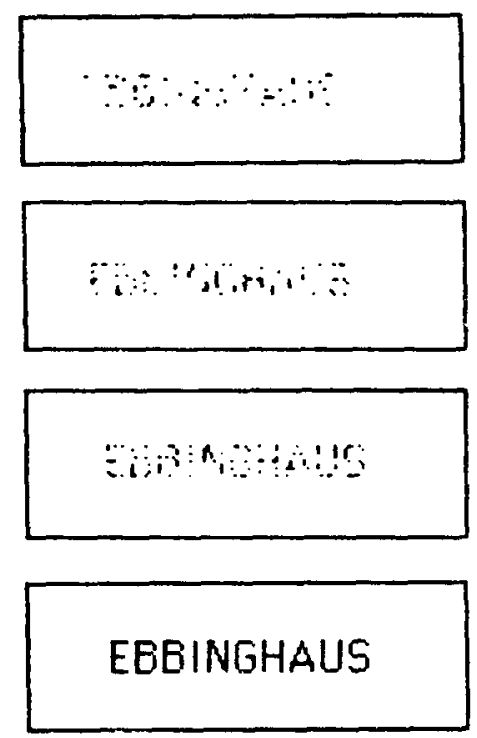

Figure 2. Three passible steps in the materialization of the word Ebbinghaus.

and made available simply by including BASIC's LIBRARY statement at the beginning of the program. Any routine in the library can be used with a single statement, just as you would use any BASIC command. Therefore, no knowledge of assembly language is required to use the routines described in this paper, which are contained in a machine language library called PIDLib. Appendix A contains the syntax for each routine. The hex codes are provided in Appendix B so that programmers may enter the code segments directly into their libraries using programs such as ResEdit. The code for LibInit is included so that new libraries may be created.

\section{THE ROUTINES}

\section{Bit Images}

The stimuli and masks for the perceptual identification routines must be in the form of bit images. A bit image is a collection of bits in memory that represent the pixels of a graphic image on a one-to-one basis. Because of the unique way in which the Macintosh handles text and graphics, anything that can be displayed on the screen, including text, can be placed into a bit image. In MSBASIC, bit images are created with the GET statement, which places the bit image into a numerical array. The GET statement is the easiest way to create a bit image of a stimulus within BASIC. Once a bit image has been created, it may be displayed with BASIC's PUT statement or with one of the perceptual identification routines in PIDLib. The presentation routines in PIDLib require that the width of a bit image be a multiple of 16 pixels. For example, a bit image with an upper left coordinate of $(20,25)$ and a lower right coordinate of $(35,45)$ is 16 pixels wide $[16=(35-20)+1]$.
Graphic stimuli on the Macintosh may be prepared in many ways, including commercially available digitizers or graphics editors such as SuperPaint or MacDraw. Stimuli created in another application can be moved into BASIC via the clipboard and the Scrapbook. This technique is rather complicated but sometimes necessary. A more detailed description of how images are transferred into MS-BASIC from other applications can be found in Brooks (1985).

\section{Masks}

The first step in generating a mask is to determine which pixels will constitute the mask. If pixels in a bit image are thought of as being numbered from left to right, top to bottom, then numbers can be assigned to the pixels that are part of the mask. The pixels that constitute the mask are designated by placing the corresponding pixel numbers in an integer array.

The PIDLib routines Assign, Shuffle, ${ }^{1}$ ClaimPixels, and CreateMask provide a way to create a random-dot mask. The Assign routine generates a series of integers and places them into an array. If a mask of 80 pixels is desired, then 80 elements of the array should be assigned values. Shuffle (Lane \& Ashby, 1987) shuffles the array and ClaimPixels claims the pixels to be in the mask by reassigning the appropriate numbers to the elements of the shuffled array. Shuffle should be called again before stimulus clarification or materialization to randomize the order of the pixel numbers contained in the array.

The second method of creating a bit image of a mask results in a reduced mask that contains only the pixels common to the mask and the stimulus. Once again, the routines Assign, Shuffle, and ClaimPixels are used to generate a random assortment of pixels that make up the mask. A reduced mask may then be created with the routine CreateRedMask. If the resulting mask is combined with the stimulus using an exclusive-OR operation then only some pixels of the stimulus would be black and no extraneous pixels would appear, yielding images similar to those in Figure 2. Thus, as a result of the exclusiveOR operation, some of the black pixels of the stimulus are changed to white.

\section{Superimposition}

Next, the bit image of the mask may be superimposed over the bit image of the stimulus. One method of superimposition requires the use of the logical operator $O R$. Although it is possible to OR two arrays with a FORNEXT loop in BASIC, it is time consuming: To OR a 5,000 -element array takes about $17 \mathrm{sec}$. The PIDLib routine called BlendOR serves the same function but is much faster: To OR a 5,000-element array takes less than $0.2 \mathrm{sec}$. The BlendOR routine returns the bit image of the resulting degraded stimulus in an array. An example of the result of such an operation is provided in Figure 3A. Similarly, a logical exclusive-OR (XOR) may be per- 

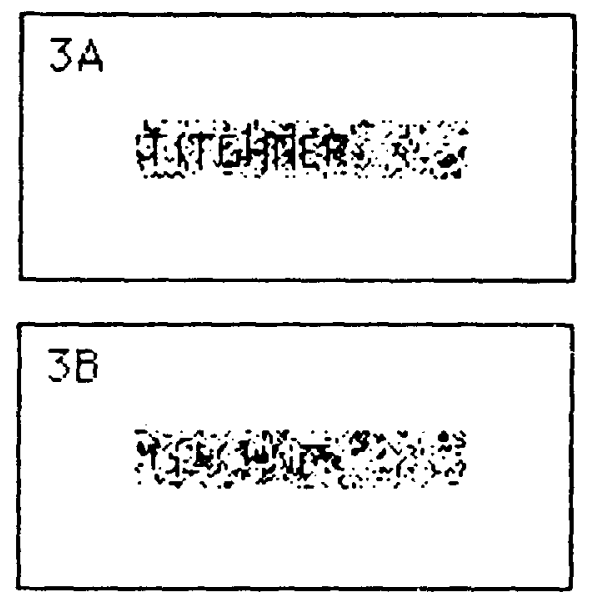

Figure 3. Panel 3A illustrates a stimulus that has been combined with a mask using a logical-OR operation. Panel 3B illustrates the same stimulus combined with a mask using an exclusive-OR operation.

formed with equal speed on two arrays with the routine BlendXOR to obtain a degraded stimulus, as shown in Figure 3B, in which some of the black pixels of the stimulus have been turned white. The BlendOR and BlendXOR routines may be used to combine any two integer arrays.

After a mask and a stimulus have been combined, the routine DefineMask should be used to determine which pixels are actually black in the bit image of the mask. The numbers of the black pixels are placed in another integer array. The number of black pixels in the mask is also provided by this routine. The purpose of DefineMask is to allow the Clarify routine, which relies on an iterative process, to function smoothly and rapidly.

\section{PERCEPTUAL IDENTIFICATION}

\section{Stimulus Clarification}

Stimulus clarification through the stepwise removal of a mask is accomplished with the PIDLib routine called Clarify. This routine allows the specification of the rate and order of removal of the pixels in the mask. The pixels may be removed at any rate that is a multiple of $16.63 \mathrm{msec}$, the rate at which the Macintosh screen is refreshed. The Clarify routine removes pixels from the mask until the mask disappears or until the subject makes a keyboard response. This routine removes masks that have been combined with stimuli using either BlendOR or BlendXOR. Alternatively, the Clarify routine may be embedded in a FOR-NEXT loop and pixel removal may be paced by the subject or the experimenter.

The Clarify routine returns, in an integer array, the keyboard response and the amount of time elapsed since the first degraded image was displayed. Either of these measures could serve as a dependent variable.

\section{Stimulus Materialization}

Displaying the stimulus on the screen pixel by pixel in an incremental fashion (stimulus materialization) is also accomplished with the Clarify routine. The factor that determines whether a stimulus is materialized or clarified is the manner in which the mask was created. For stimulus materialization, the mask must be created with the CreateRedMask routine.

\section{Demonstration Experiment}

A demonstration experiment was conducted in which 16 subjects read aloud alist of 48 words. Each word was presented for $1,663 \mathrm{msec}$ with a 2,000 -msec interval between successive words. The identification phase consisted of 96 words, half of which had been presented previously and half of which had not. Half of the new words and half of the old words were degraded with the stimulus clarification procedure. The remainder of the words were degraded with the stimulus materialization procedure. Therefore, 48 words were degraded under each procedure. As expected, prior exposure greatly facilitated later identification of the words $[t(15)=5.57, p<.0001]$. For stimulus materialization, subjects required $7,393 \mathrm{msec}$ to identify the new words and 6,856 msec to identify the old words. The identification latencies were slightly longer for the stimulus clarification task: $9,300 \mathrm{msec}$ for new words and 9,074 msec for old words.

\section{SAMPLE PROGRAMS}

The sample program in Listing A illustrates the use of stimulus clarification. In this program a 1536-pixel mask is superimposed over a 1536-pixel bit image of a word. First, the LIBRARY statement is used so that the routines in PIDLib can be called. Pixels are then randomly selected so that $75 \%$ of the pixels in the mask are black. CreateMask is used to create the desired bit image of the mask, which is then superimposed over the bit image of the word with BlendXOR. Finally, the stimulus is clarified with the Clarify routine at the rate of 1 pixel per $16.63 \mathrm{msec}$.

A program that uses stimulus materialization is provided in Listing B. In this program the stimulus is once again the 1563-pixel bit image of a word, but the mask is created with CreateRedMask. The Clarify routine places one pixel of the stimulus at a time on the screen at the rate of 1 pixel per $99.78 \mathrm{msec}$.

The PIDLib routine called DefineMask determines the numbers of the black pixels in the mask and places them into an integer array. The number of pixels in the mask is placed into the variable actual $\%$. The value of actual\% determines how many pixels will be removed from the mask. Therefore, if actual\% contains the number of pixels in the mask, the mask will be removed entirely as a result of the Clarify routine. If, on the other hand, the value of actual\% is less than the number of pixels in the mask, the mask will not be removed completely.

In both programs, the information that is returned in the array record $\%$ is equivalent: The key that was pressed and the amount of time elapsed since the routine began its presentation. Both of the sample programs create the bit image of the word for illustrative purposes. Normally, 
bit images would be created at some time prior to the experiment and placed in a file for later use.

\section{AVAILABILITY}

A disk containing the machine language library PIDLib and the instructions may be obtained by sending $\$ 6$ to the author.

\section{REFERENCES}

Brooks, J. O., III. (1985). Pictorial stimuli for the Apple Macintosh computer. Behavior Research Methods, Instruments, \& Computers, 17, 409-410.

Cermak, L. S., Talbot, N., Chandler, K., \& Wolbarst, L. R. (1985). The perceptual priming phenomenon in amnesia. Neuropsychologia, 23, 615-622.
GoLuIN, E. S. (1960). Developmental studies of visual recognition of incomplete objects. Perceptual \& Motor Skills, 11, 289-298.

Johnston, W. A., DARK, V. J., \& JACOBY, L. L. (1985). Perceptual fluency and recognition judgments. Journal of Experimental Psychology: Learning, Memory, \& Cognition, 11, 3-11.

LANE, D. L., \& AshBY, A. B. (1987). PsychLib: A library of machinelanguage routines for controlling psychology experiments on the Apple Macintosh computer. Behavior Research Methods, Instruments, \& Computers, 19, 246-248.

Vokey, J. R., BAKER, J. G., HAYman, G., \& JACOBY, L. L. (1986). Perceptual identification of visually degraded stimuli. Behavior Research Methods, Instruments, \& Computers, 18, 1-9.

\section{NOTE}

1. The Shuffle routine was written by Lane and Ashby (1987) and is included in PIDLib with their permission.

\section{LISTING A}

\section{LIBRARY "PIDLIb" OPTION BASE 1}

numpix $\%=1536$ 'the number of pixels in the bit images

DIM pixInfo\%(numpix\%),masklmage $\%(500)$,stimlmage $\%(500)$

DIM degrade\%(500),rec\%(5)

$75 \%$ of the pixels will be black

black\%=numpix\% * .75

DIM Mdef\%(black\%)

Assign! pixlnfo\%(1),black\%

'randomizing the order with the Shuffle routine (Lane \& Ashby, 1986)

Shuffle! numpix\%,pixInfo\%(1)

'reclaiming the pixels that are black

ClaimPixels! pixInfo\%(1),numpix\%

'randomizing the order for the Clarify routine

Shuffle! numpix\%,pixInfo\%(1)

'Setting up the bit images from a blank screen

\section{CLS}

GET $(10,10)-(105,52)$,masklmage $\%$

MOVETO $(14,30)$ : PRINT "Ebbinghaus"

GET $(10,10)-(105,52)$,stimlmage $\%$

CLS

'Creating the mask

CreateMask! pixInfo\%(1),masklmage\%(1),numpix\% 
'Superimposing the mask on the stimulus. The result is returned 'in degrade\%

BlendXOR! 500,masklmage $\%(1)$,stimlmage $\%(1)$,degrade $\%(1)$

'Defining the mask

actual $\%=0$

'all variables must be defined before using

DefineMask! pixlnfo\%(1),masklmage $\%(1)$, Mdef\%(1),numpix\%,actual\%

'now Mdef\% contains the numbers of the black pixels in the mask

'Waiting for key press to begin

LOCATE 10,20: PRINT "Hit any key to begin"

$z \$=I N K E Y \$:$ WHILE $2 \$=" n: z \$=I N K E Y \$:$ WEND

'Removing the mask
mode $\%=1$
'mode $\%=1$ for BlendXOR
'mode $\%=1$ for BlendOR
rate $\%=1$
'rate of pixel removal = 1 pixel $/ 60$ th sec.
rec $\%(1)=1$
'stop clarification after 1 keypress
$x \%=200: y \%=200$
'coordinates of the image on the screen

Clarify! rec $\%(1)$, mode $\%, x \%, y \%$, rate $\%$, masklmage $\%(1)$, Mdef $\%(1)$, degrade $\%(1)$, actual $\%$

\section{LISTING B}

\section{LIBRARY "PIDLIb" \\ OPTION BASE 1}

numpix $\%=1536$

'the number of pixels in the bit images

DIM pixInfo\%(numpix\%),masklmage $\%(500)$,stimimage $\%(500)$

DIM degrade\%(500), rec\%(5)

$75 \%$ of the pixels will be black

black $\%=$ numpix $\%$ *.75

DIM Mdef\%(black\%)

Assign! pixlnfo\%(1),black \%

'randomizing the order with the Shuffle routine (Lane \& Ashby, 1986)

Shuffle! numpix\%,pixlnfo\%(1)

'reclaiming the pixels that are black

ClaimPixels! pixlnfo\%(1),numpix\%

'randomizing the order for the Clarify routine

Shuffle! numpix\%,pixlnfo\%(1) 
'Setting up the bit images

\section{CLS}

GET $(10,10)-(105,52)$, maskImage $\%$

MOVETO $(14,30)$ : PRINT "Ebbinghaus"

GET $(10,10)-(105,52)$,stimlmage\%

\section{CLS}

'Creating the mask

CreateRedMask! pixInfo\%(1),masklmage $\%(1)$,stimlmage $\%(1)$,numpix $\%$

'Superimposing the mask on the stimulus. The result is returned 'in degrade $\%$

BlendXOR! 500,masklmage $\%(1)$, stimlmage $\%(1)$,degrade $\%(1)$

'Defining the mask

actual $\%=0$

'all variables must be defined before using

DefineMask! pixInfo\%(1),masklmage $\%(1)$, Mdef\%(1), numpix $\%$,actual\%

'now Mdef\% contains the numbers of the black pixels in the mask

'Waiting for key press to begin

LOCATE 10,20: PRINT "Hit any key to begin"

$z \$=I N K E Y \$:$ WHILE $z \$={ }^{n n}: z \$=I N K E Y \$:$ WEND

'Removing the mask

mode $\%=1$

rate $\%=6$

rec $\%(1)=1$

$x \%=200: y \%=200$ 'mode $\%=1$ for BlendXOR

'mode $\%=1$ for BlendOR

'rate of pixel removal = 1 pixels each

'6/60th sec.

'stop clarification after 1 keypress

'coordinates of the image on the screen

Clarify! rec\%(1),mode $\%, x \%, y \%$,rate $\%$,maskimage $\%(1)$, Mdef $\%(1)$, degrade $\%(1)$,aclual $\%$

\section{APPENDIX A}

\section{GENERAL NOTES:}

A variable must be defined betore it is passed to a routine. The exclamation point should follow the names of all routines. Be sure to pay close attention to the types of the variables.

If the argument is not an array, the value does not have to be placed in a variable. For example, if $n \%=10$ then the following two statements produce equivalent results: 


$$
\text { Assign! array\%(1),10 }
$$

Whenever an argument is an array, the element of the array that is passed determines the starting point of the operations. For most purposes, the first element of the array should be passed to the routine.

Assign! array $\%(1), n \%$

This statement places a series of integers trom 1 to $n \%$ in array\%. It is equivalent to, and much faster than, the following MS-BASIC statements: FOR $i=1$ to $n \%:$ array $\%(i)=i:$ NEXT $i$

BlendOR! size $\%$,masklmage $\%(1)$,stimuluslmage $\%(1)$,degradedlmage $\%$ (1) size $\%$ is the number of elements in the three arrays. This routine performs a logical or using the arrays masklmage $\%$ and stimuluslmage $\%$ and places the result in the array degradedimage $\%$. If size $\%$ is equal to the size of the arrays and the first element of each array is passed to BlendOR then all elements of the arrays will be blended. If only masklmage\%(10) through masklmage $\%(20)$ are to be blended with stimuluslmage\%(1) through stimulusImage\%(10), then the command would read as follows: BlendOR! 10 ,masklmage $\%(10)$,stimuluslmage $\%(1)$,degradedlmage $\%(1)$

BlendXOR! size\%,masklmage \%(1),stimulusImage $\%(1)$,degradedlmage $\%(1)$

This routine is the same as BlendOR except that it performs a logical exclusive-or on the two arrays.

ClaimPixels! pixellnfo\%(1),numelements $\%$

This routine "reclaims" pixels that have been shuffled in the array pixellnfo\%. This is equivalent to the following BASIC statements:

FOR $i=1$ to numelements $\%$

IF pixellnfo\%(i) $<>0$ THEN pixellnfo\%(i) = i

NEXT i

Clarify!

rec\%(1),mode $\%, x \%, y \%$, rate $\%$, Image $1 \%(1)$, Def\%(1),Image $2 \%(1)$, actual\%

This routine is best explained by the arguments it takes:

rec\%(1) - the maximum number of responses that can occur. The value of 


\section{APPENDIX A (Continued)}

this element must be set to a nonzero value before Clarify is called.

For a typical perceptual identification experiment, rec\%(1) should be set to 1 . If rec\%(1) is greater than 1 then rec\%(2) through rec\%(5) are repeated for each response. Note that the routine returns the values of rec\%(2) through rec\%(5).

rec\%(2) - the type of response that occurred.

$$
\begin{aligned}
& 0 \text { - null } \\
& 1 \text { - mouse was pressed down } \\
& 3 \text { - key press }
\end{aligned}
$$

rec\%(3) - the elapsed time in 60th's of a second rec\%(4) - if a mouse click occurred, this is the $x$-coordinate of the cursor. If a key was pressed, then the ASCII code is returned rec\%(5) - if the response was a mouse click, then this element contains the $y$-coordinate of the cursor.

mode\% - determines the manner of pixel removal. Mode\% should equal 1 for images that were blended with BlendXOR or 0 for images that were created with BlendOR .

$x \%, y \%$ - the coordinate at which the upper left corner of the bit image will be displayed.

rate $\%$ - the rate of pixel removal or addition in screen refreshes $(60.13 \mathrm{~Hz})$. If rate\% equals 1 then 1 pixel is removed every $16.63 \mathrm{~ms}$. If rate\% equals 3 then 1 pixel is removed every $49.89 \mathrm{~ms}$.

Image $1 \%(1)$ - the first element of the bit image of the mask that was blended with the bit image of the stimulus. If mode\% equals 0 , then Image $1 \%(1)$ should be the first element of the bit image of the stimulus.

Def\%(1) - the values in this array were returned from the DefineMask routine. Def\% contains the numbers of the pixels in the mask. Image $2 \%(1)$ - the first element of the bit image of the degraded stimulus. actual\% - contains the number of pixels that will be removed from the mask if the subject makes no response.

CreateMask! pixInfo\%(1),maskImage\%(1),numpix\% pixinfo\% is the array containing the numbers of the pixels that will be black 
in the bit image of the mask (Masklmage\%). numPix\% is the number of elements in pixinfo\%.

CreateRedMask! pixInfo\%(1),Masklmage \%(1),Stimlmage\%(1),numpix\%

This routine is similar to CreateMask except that it creates a mask containing only those pixels common to both the mask and the stimulus.

MaskImage\%(1) and Stimlmage $\%(1)$ are the bit images of the mask and stimulus, respectively.

DefineMask! pixinfo\%(1), Masklmage\%(1),MDef\%(1),numelem\%,actual\%

The numbers of the black pixels in the mask are contained in pix 1 nfo\%.

The bit image of the mask is in Masklmage\%. Mdef\% is an array that will contain a compressed version of pix Info\%. numelem $\%$ is the number of elements in pixInfo\%. The variable actual\% will contain the number of black pixels in the mask.

Shuffle! $n \%$,array\%(1)

This routine shuffles the first $n \%$ elements of array\% beginning with array $\%(n)$. For example, if elements array $\%(10)$ through array\%(20) are to be shutfled, then the statement would read Shuffle! 10,array\%(10).

\section{APPENDIX B}

\begin{tabular}{lllllllll}
\multicolumn{10}{c}{ Assign } \\
00 & 00 & 00 & 01 & $4 E$ & $A D$ & 00 & $2 A$ \\
$O C$ & 40 & 00 & 03 & & 66 & 30 & 26 & $4 A$ \\
$4 E$ & $A D$ & 00 & $2 A$ & & 08 & 80 & 00 & $O F$ \\
$0 C$ & 40 & 00 & 03 & & 66 & 20 & $3 C$ & 12 \\
34 & 06 & 53 & 42 & & $D 6$ & $C 2$ & $D 6$ & $C 2$ \\
60 & $0 C$ & $3 A$ & 06 & & 52 & 45 & 36 & 85 \\
97 & $F C$ & 00 & 00 & & 00 & 02 & 51 & $C E$ \\
FF & F2 & 70 & 00 & & $4 E$ & 75 & 74 & $O D$ \\
70 & 00 & $4 E$ & $A D$ & & 00 & 42 & &
\end{tabular}

00000001 BlendOR

$08 \quad 8000 \quad 0 F \quad 0 C 4000 \quad 03$

$\begin{array}{lllllllll}66 & 60 & 3 \mathrm{C} & 12 & 55 & 46 & 4 \mathrm{E} & \mathrm{AD}\end{array}$

$00 \quad 2 A O C \quad 40 \quad 00036652$

26 AA D7 EC $\quad 00000004$

$4 E A D O 02 A \quad O C 40 \quad 0003$

$\begin{array}{lllll}66 & 40 & 28 & 4 A & \text { D }\end{array}$ FC $00 \quad 00$

$00 \quad 04 \quad 4 E$ AD $\quad 00 \quad 2 A \quad 0 C 40$

$000366 \quad 2 E$ DS FC $00 \quad 00$

$00043 A 13 \quad 39148445$

$34 \quad 82$ D7 FC $\quad 00000002$

09 FC $0000 \quad 0002$ D5 FC

$0000 \quad 0002 \quad 5346$ OC 46

$00006702 \quad 60$ DC 7000

$4 E 7574 O D \quad 70 \quad 00 \quad 4 E \quad A D$

0042

\section{BlendYOR}

00000001

$08 \quad 80000 \%$

$\begin{array}{lllll}66 & 60 & 3 C & 12\end{array}$

$00 \quad 2 A$ OC 40

26 4A D 7 FC

$4 E \quad A D \quad 00 \quad 2 A$

$\begin{array}{lllll}66 & 40 & 28 & 4 A\end{array}$
OC $40 \quad 0003$

$\begin{array}{lllll}55 & 46 & 4 E & A D\end{array}$

$\begin{array}{lllll}00 & 03 & 66 & 52\end{array}$

$\begin{array}{llll}00 & 00 & 00 & 04\end{array}$

OC $40 \quad 0003$

D9 FC 0000
$4 E \quad A D \quad 00 \quad 2 A$
0004 4E AD

$\begin{array}{lllll}00 & 03 & 66 & 2 \mathrm{E}\end{array}$

$\begin{array}{lllll}00 & 04 & 3 A & 13\end{array}$

$\begin{array}{llll}34 & B 2 & D 7 & F C\end{array}$

D9 FC 0000

$\begin{array}{lllll}00 & 00 & 00 & 02\end{array}$

$\begin{array}{llll}00 & 00 & 67 & 02\end{array}$

$4 E 75 \quad 740 D$

0042

$$
\text { Claimpixels }
$$

$000800 \quad 00$

OC $40 \quad 00 \quad 03$

4 E AD $00 \quad 2 \mathrm{~A}$

OC $40 \quad 00 \quad 03$

$\begin{array}{lllll}60 & 14 & 3 A & 06\end{array}$

OC $4400 \quad 00$

D7 FC 0000

FE EA $70 \quad 00$

$\begin{array}{llll}70 & 00 & 4 E & A D\end{array}$

2A OC 40

D5 FC $00 \quad 00$

$\begin{array}{lllll}34 & 14 & B B & 42\end{array}$

00000002

0002 D5 FC

$\begin{array}{lllll}53 & 46 & O C & 46\end{array}$

60 DC $70 \quad 00$

$70 \quad 00 \quad 4 E \quad A D$

$70 \quad 00 \quad 4 E A$

$$
\text { Clarify }
$$

$0000 \quad 0001$

$27 \quad 4 F \quad F F \quad E 6$

32 BC $00 \quad 00$

7200049 FA

$O O \quad 2 A \quad O C \quad 40$

02 5C 2C $4 A$

27 TE FF DE

34 9E $4 E$ AD

OO OF OC 40

$02 \quad 3 C \quad 3752$

00 2A $08 \quad B 0$

$\begin{array}{lllll}00 & 03 & 66 & 00\end{array}$

$\begin{array}{llll}00 & 02 & 4 E & A D\end{array}$

OO OF OC 40

$02 \quad 14$ OC 40

$4 E$ AD $00 \quad 2 \mathrm{~A}$

$\begin{array}{lllll}66 & 30 & 26 & 4 \mathrm{~A}\end{array}$ $0 B 80 \quad 00 \quad O F$ $\begin{array}{llll}66 & 20 & 30 & 12\end{array}$ $\begin{array}{llll}52 & 45 & 38 & 13\end{array}$ $\begin{array}{llll}67 & 02 & 36 & 85\end{array}$ $000251 \mathrm{CE}$ $4 E 7574$ OD 0042

4E 53 FE DC 43 EA 0298 43 EA $027 C$ 02 7A 4E AD $\begin{array}{llll}00 & 03 & 66 & 00\end{array}$ 27 4E FF EA 45 FA 0276 $00 \quad 2 A 08 \quad B 0$ $\begin{array}{lllll}00 & 03 & 66 & 00\end{array}$ FF $D C$ AE $A D$ OO OF OC 40 $\begin{array}{llll}02 & 28 & 33 & 52\end{array}$ $00 \quad 2 A \quad 08 \quad 80$ $\begin{array}{llll}00 & 03 & 66 & 00\end{array}$ $00 \quad 00 \quad 66 \quad 0 A$ 
APPENDIX B (Continued)

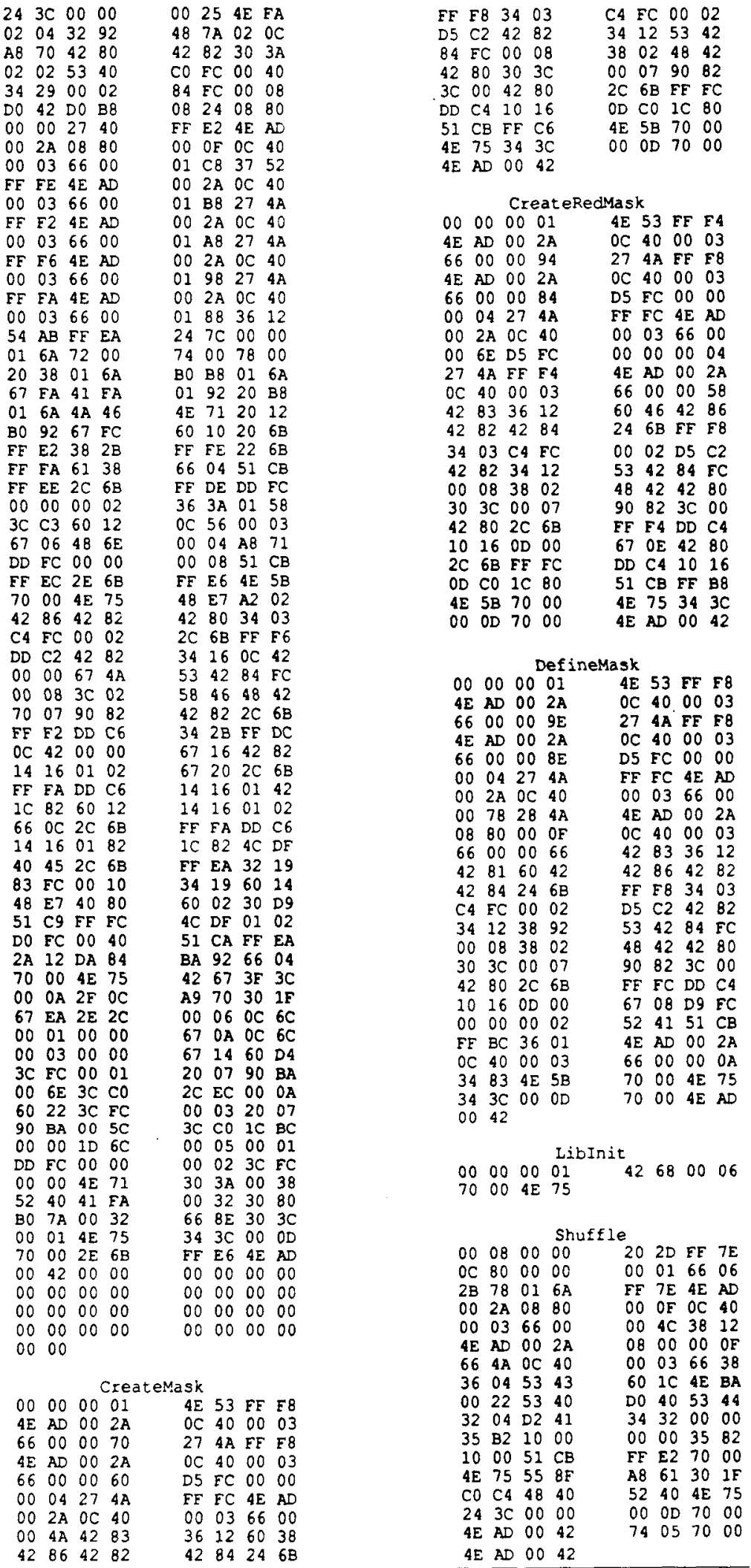

